\title{
OPEN Unravelling the intricate cooperativity of subunit gating in P2X2 ion channels
}

\author{
Christian Sattler ${ }^{1,4}$, Thomas Eick ${ }^{1,4}$, Sabine Hummert ${ }^{1}$, Eckhard Schulz ${ }^{2}$, Ralf Schmauder ${ }^{1}$, \\ Andrea Schweinitz ${ }^{1}$, Christopher Unzeitig ${ }^{1}$, Frank Schwede ${ }^{3}$ \& Klaus Benndorf ${ }^{1 凶}$
}

Ionotropic purinergic (P2X) receptors are trimeric channels that are activated by the binding of ATP. They are involved in multiple physiological functions, including synaptic transmission, pain and inflammation. The mechanism of activation is still elusive. Here we kinetically unraveled and quantified subunit activation in P2X2 receptors by an extensive global fit approach with four complex and intimately coupled kinetic schemes to currents obtained from wild type and mutated receptors using ATP and its fluorescent derivative 2-[DY-547P1]-AET-ATP (fATP). We show that the steep concentration-activation relationship in wild type channels is caused by a subunit flip reaction with strong positive cooperativity, overbalancing a pronounced negative cooperativity for the three ATP binding steps, that the net probability fluxes in the model generate a marked hysteresis in the activation-deactivation cycle, and that the predicted fATP binding matches the binding measured by fluorescence. Our results shed light into the intricate activation process of $\mathrm{P} 2 \mathrm{X}$ channels.

Ionotropic purinergic receptors (P2X receptors) are expressed in many tissues and they are involved in diverse physiological and pathophysiological processes like pain, inflammation, taste or synaptic transmission ${ }^{1,2}$. The channels are activated by the binding of ATP at their extracellular side and they generate a non-specific cation conductance ${ }^{3}$. Seven mammalian subunit isoforms have been identified ${ }^{4,5}$. The crystal structure of the zebrafish $\mathrm{P} 2 \mathrm{X} 4$ (zfP2X4) channel has proven a trimeric architecture and revealed that the shape of a single subunit resembles that of a dolphin ${ }^{6,7}$, which was later confirmed for P2X3 receptors ${ }^{8}$. The transmembrane domains TM1 and TM2 form the tail and the ensemble of the three TM2 helices in a channel builds the pore ${ }^{9}$. The large ectodomain is organized in a $\beta$-sheet structure with lateral fenestration sites. From this body four structurally flexible domains ramify, including head, dorsal fin, left and right flipper. ATP is bound to binding sites between two neighbored subunits, distant by as much as $40 \AA$ from the extracellular boundary of the transmembrane domain, and cradled by the dolphin head, upper body, lower body, left flipper and dorsal fin.

It is presently not fully clear how the signal of ligand binding propagates and opens the channel pore. Two studies suggested that two activated subunits suffice for channel activation ${ }^{10-12}$ whereas other studies provide evidence that all three subunits are involved ${ }^{10,13-15}$. For transmission the signal of ATP binding to the pore opening, a central role of the $\beta$ - 14 sheet has been suggested, connecting within a subunit the ATP binding site on the upper end with the pore forming TM2 helix on the lower end, thereby interacting with the $\beta-1$ sheet of the adjacent subunit ${ }^{16}$. Regarding the subunit interaction, it has been proposed that each ATP-binding signal propagates first along the same subunit, then spreads equally to all three subunits towards the pore ${ }^{11}$ and results in a symmetrical closed-open transition ${ }^{17}$. Another study distinguishes five key steps in activation ${ }^{18}$. However, many questions remain open, including the type of subunit cooperativity and propagation of ligand binding to the opening of the gate.

Major progress in understanding the function of P2X receptors can be expected from applying global fit analyses of extended data, as demonstrated previously for other ion channels. Using current data at equilibrium this has been demonstrated for concentration-activation relationships of concatenated CNGA2 channels, containing a different number of disabled binding sites ${ }^{19}$, for $I V$-relationships of viral Kv channels at different concentrations of permeating $\mathrm{K}^{+}$ions ${ }^{20}$, or gating charge movement in Shaker channels ${ }^{21}$. We previously also globally fitted data distant from equilibrium by kinetic schemes, for activation time courses of tetrameric CNGA2 channels induced

${ }^{1}$ Institut für Physiologie II, Universitätsklinikum Jena, Friedrich-Schiller-Universität Jena, Kollegiengasse 9, 07743 Jena, Germany. ${ }^{2}$ Fakultät Elektrotechnik, Fachhochschule Schmalkalden, Blechhammer, 98574 Schmalkalden, Germany. ${ }^{3}$ BIOLOG Life Science Institute GmbH \& Co. KG, Flughafendamm 9A, 28199 Bremen, Germany. ${ }^{4}$ These authors contributed equally: Christian Sattler and Thomas Eick. ${ }^{\boxplus e m a i l:}$ Klaus.Benndorf@med.uni-jena.de 
by cGMP jumps to different concentrations ${ }^{22}$ and voltage-induced activation time courses of tetrameric HCN2 channels ${ }^{23}$. Moreover, we extended these analyses by combining electrophysiological time-dependent data with the corresponding orthogonal data of ligand binding which enabled us to specify kinetic schemes in considerable detail and to identify complex types of cooperativity among the subunits in CNGA2 ${ }^{24,25}$ and HCN2 channels ${ }^{26}$.

Herein we analyzed ATP-induced activation of P2X2 receptors in great detail by a novel global fit approach with extended and orthogonal data. We included both equilibrium and non-equilibrium data at multiple ligand concentrations, data from the facilitating mutant $\mathrm{H} 319 \mathrm{~K}$ in the transmission pathway, and data obtained with an ATP-derivative with significantly lower apparent affinity 2-[DY-547P1]-AET-ATP (fATP) ${ }^{27}$. We globally fitted these heterogeneous data with four complex kinetic schemes, each including three binding reactions, three flip and three open reactions and, importantly, the four models were intimately coupled by sharing the majority of parameters. These results led us identify and quantify a simple, and also surprising, cooperativity pattern for the wild type channels and recognize that the steep concentration-activation relationship in P2X2 channels is the result of a subunit flip reaction with pronounced positive cooperativity that overbalances pronounced negative cooperativity for the three ATP binding steps. Moreover, it is shown how the mutation H319K promotes opening by significantly facilitating the conformational flip in the channel liganded by only one ATP.

\section{Results}

Open probability in macroscopic currents. All recordings subjected to the global fit analysis were performed in whole HEK293 cells at $-50 \mathrm{mV}$ to stay in the physiological range of voltages and to obtain currents of reasonable amplitude. Solution jumps were administered by a fast application system, enabling the application of multiple concentrations to the same cell. The evoked currents were activated within hundreds of milliseconds to seconds and deactivated when jumping back to control solution (Fig. 1a). Slow desensitization in the presence of ATP, mostly pronounced at high ligand concentrations, was eliminated by including only the currents until the time of peak. The peak currents were related to those at saturating ATP to obtain the normalized concentrationactivation-relationships (Fig. 1a; see "Methods").

For the modeling below, the value of the open probability at saturating ATP, $P_{\mathrm{o} \text {, max }}$, was required to translate all ensemble currents to time courses of the open probability, $P_{\mathrm{o}}$, at the actual ATP concentration. $P_{\mathrm{o} \text {, max }}$ was determined from evaluating the noise variance $\sigma^{2}$ near the peak current $I_{\max }$ at saturating ATP, which was corrected by the variance of the baseline, according to

$$
P_{o, \max }=1-\sigma^{2} / i I_{\max } .
$$

Herein, $i$ is the amplitude of the single-channel current that was determined experimentally. The value was 0.80 $\mathrm{pA} \pm 0.03 \mathrm{pA}(\mathrm{n}=6)($ Fig. $1 \mathrm{~b})$, resulting in a $P_{\mathrm{o}, \max }$ value of $0.695 \pm 0.009(\mathrm{n}=6)$. With this value the concentrationactivation relationship was translated to a concentration- $P_{\mathrm{o}}$ relationship to be used in the global fit approach.

Model selection. We considered only models with three functionally relevant binding steps because (1.) the structure reveals three ATP binding sites, located in the large extracellular domain distant from the gate in the TMD (Fig. 2a), and (2.) P2X2 concatamers with selectively disabled binding sites show a proportional increase of the current density with the number of functional binding sites ${ }^{10}$. Accordingly, our initial model comprised three binding steps followed by an open step at each degree of ligand binding (model 1; Supplementary Fig. 1a). Furthermore, for the unliganded channel we assumed that no opening proceeds because there is no experimental evidence for relevant channel activity in the absence of ATP. Since it is a priori not clear to what extent the subunits interact upon activation, fixed stoichiometries of the rate constants, statistically weighted according to the number of associating or dissociating ligands, were avoided. Model 1 with the direct closed-open transitions clearly failed to describe the data: It generated about five times slower activation time courses at intermediate concentrations compared to the measurements. Moreover, the fit produced a pronounced upward deflection in the early deactivation time courses that was not present in the data.

To overcome these mismatches, we extended our model by introducing flipped states in the closed-open isomerisations (Model F1; Fig. 2b). This extension improved the fit significantly in both the activation time courses and early deactivation (Fig. 2c,d). Because ligand binding appears distant from the channel gate (Fig. 2a), it seemed to us a plausible assumption to attribute the binding steps of the model to processes at the binding sites, the flipped steps to conformational changes propagating from the binding sites to the gate, and the opening step to a closed-open isomerization in the transmembrane helix TM2. However, despite the improvement of the fit, many errors of the parameters exceeded $100 \%$ (Supplementary Table 1), precluding any interpretation of the resulting rate constants. Hence, our approach to globally fit time courses and the concentration- $P_{\mathrm{o}}$ relationship at equilibrium for wild type channels and ATP is insufficient.

Extending the global fit strategy. In order to increase the constraint conditions and enhance model predictability, we gathered more functional information. We therefore included in our analysis P2X2 channels carrying the mutation $\mathrm{H} 319 \mathrm{~K}$, a mutation that has been reported to enhance the apparent affinity of $\mathrm{P} 2 \mathrm{X} 2$ channels by a factor of $40^{28}$. Naturally, the histidine, which is unique in P2X2 receptors and located in the upper part of the $\beta$-14 linker, is responsible for the positive modulation of protons on ATP induced currents via an allosteric binding site. Since the distance of $\mathrm{H} 319$ to all three binding sites on the one end and the gate on the other is similarly long and the $\beta$-14 linker is well in that part of the channel structure propagating the binding information to the pore, we assume that in our model this mutation affects the flip step (c.f. Figure 2a). Accordingly, a similar mechanism was attributed by the single amino acid P121 in the $\varepsilon$-subunit of nicotinic acetylcholine receptors $^{29}$. We further assume that in model $\mathrm{F} 1$ all three flipping rates $C_{\mathrm{x}} \rightarrow F_{\mathrm{x}}$ are accelerated by the same factor 
a

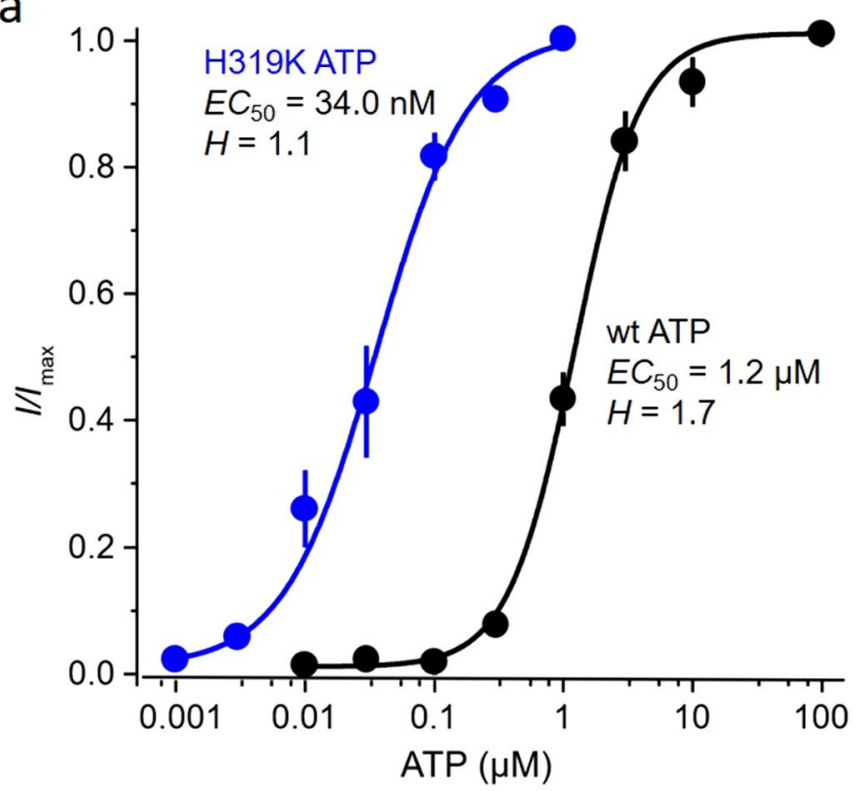

ATP

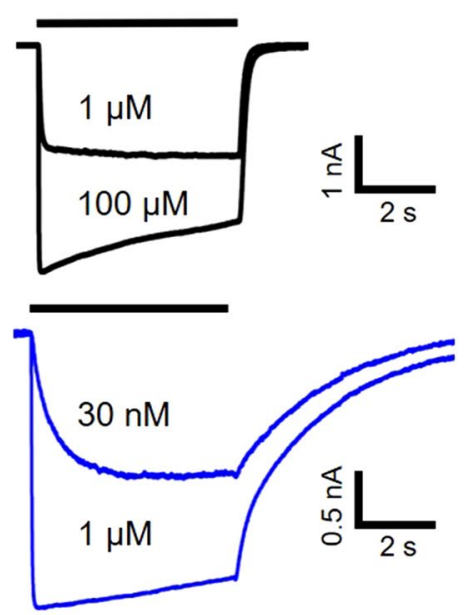

b

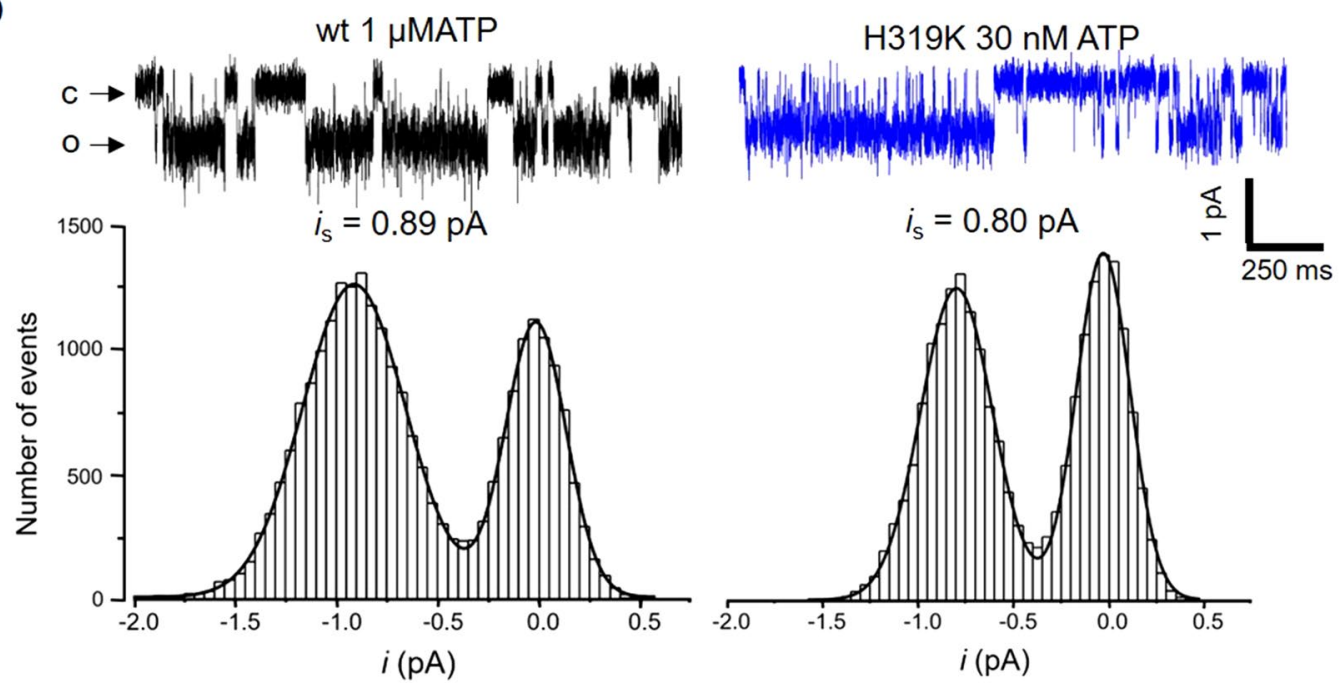

Figure 1. Currents in wild type and $\mathrm{H} 319 \mathrm{~K}$ channels. The membrane voltage was generally $-50 \mathrm{mV}$. (a) Concentration-activation relationships for wt (black) and H319K currents (blue) measured in whole cells. The data points were fitted with Hill curves according to Eq. (4) yielding the indicated parameters. The insets right show typical current time courses evoked by ATP pulses at the indicated concentrations. (b) Single-channel currents measured in the cell-attached configuration. Representative traces and amplitude histograms to determine the amplitude of the single-channel current $i_{\mathrm{s}}$ (see "Methods").

$f$ whereas the reverse reactions $F_{\mathrm{x}} \rightarrow C_{\mathrm{x}}$ are decelerated by the factor $g$, yielding model $\mathrm{F} 2$. All other rate constants in model F1 and F2 were the same (Fig. 3b; Supplementary Fig. 2). $P_{\mathrm{o} \text { max }}$ in H319K channels was determined analogue as described above for wt channels. The single-channel current amplitude was determined to be 0.78 $\mathrm{pA} \pm 0.05 \mathrm{pA}(\mathrm{n}=5)$ (Fig. 1b) which was not statistically different $(\mathrm{p}=0.66$; $t$-test) from wt channels, resulting in a $P_{\mathrm{o} \text { max }}$ value of $0.735 \pm 0.054(\mathrm{n}=7)$. For the modeling we therefore pooled the data and used the same maximum $P_{\mathrm{o}, \max }=0.719 \pm 0.031(\mathrm{n}=13)$. Then the intimately coupled models F1 and F2 were globally fitted to the two respective data sets $\left({ }^{2}\right.$ global fit). The result with ${ }^{2}$ global fit was a new set of rate constants and, notably, that the relative error of the parameters was significantly smaller than fitting only model F1 to the wt ATP data with ${ }^{1}$ global fit (Supplementary Table 2).

To further increase the experimental information, we modulated the binding process by using a second ligand with lower potency to activate the channels. We used the fluorescent 2-[DY-547P1]-AET-ATP (fATP) ${ }^{27}$ whose apparent affinity is 9 and 28 times reduced for wt and $\mathrm{H} 319$ channels, respectively. (The fact that fATP can report binding via the fluorescence intensity will allow us below to experimentally verify the calculated receptor occupancy.) For wt ATP and wt fATP we performed another ${ }^{2}$ global fit with model F1 and F3 by introducing the factor $h$ and $j$ in all binding and unbinding reactions (Fig. 3c; Supplementary Fig. 3), respectively, yielding a 
a

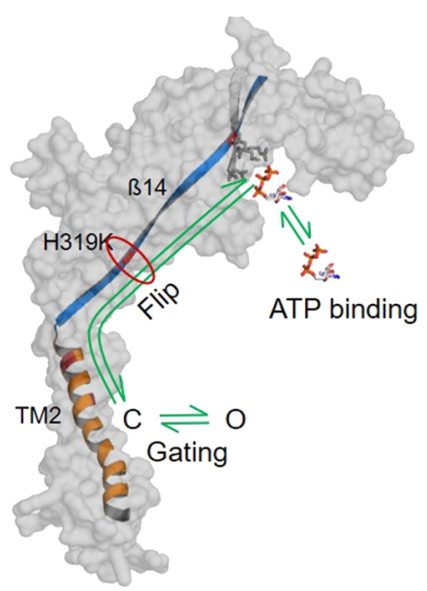

C

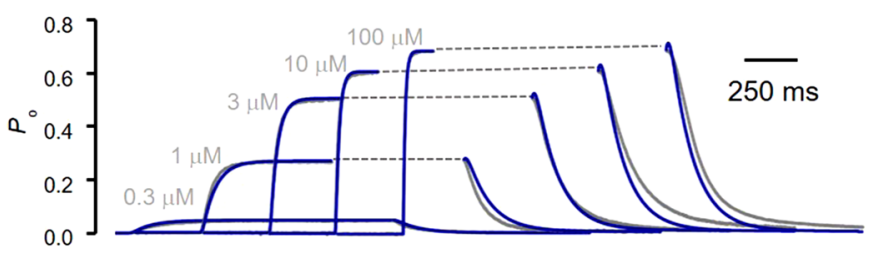

b wt ATP - model F1

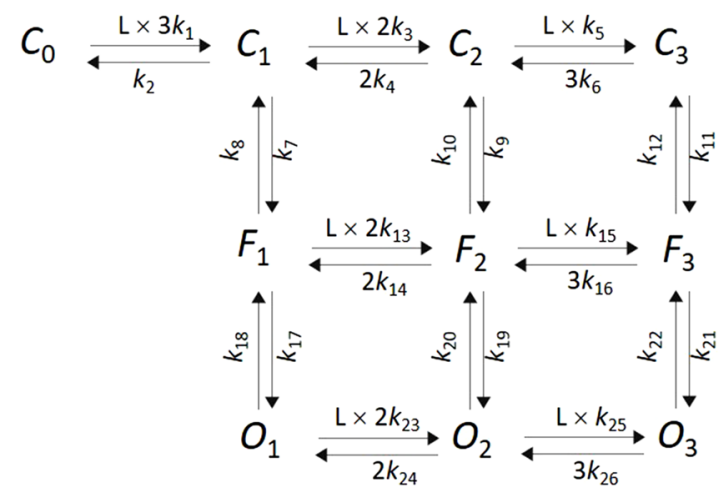

d

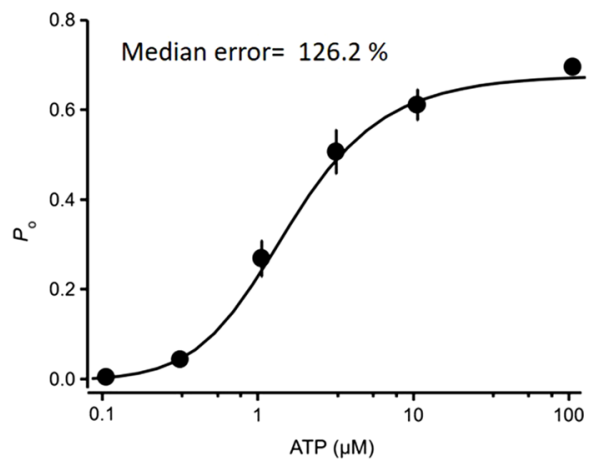

Figure 2. Global fit strategy. (a) Allocation of the processes governing the activation gating in P2X channels to the structure. As illustrated for one subunit, channel activation is composed of three steps including ATP binding, propagation of the binding signal by a conformational flip to the transmembrane gate, and gate opening. In the trimeric channel, interactions of the subunits have to be included. The subunit structure of P2X2 has been built as homology model to $\mathrm{P}_{\mathrm{XX}} 3^{8,27}$. (b) Scheme of model F1 with three binding steps, $\left(C_{\mathrm{x}-1}-C_{\mathrm{x}}\right)$, respective flip reactions, $C_{\mathrm{x}}-F_{\mathrm{x}}$, and flipped-open isomerizations, $F_{\mathrm{x}}-O_{\mathrm{x}}(\mathrm{x}=1,2,3)$, i.e. ligand binding and unbinding proceeds in horizontal direction whereas the conformational flip $\left(C_{\mathrm{x}}-F_{\mathrm{x}}\right)$ and the closed-open isomerization $\left(F_{\mathrm{x}}-O_{\mathrm{x}}\right)$ in vertical direction. $k_{\mathrm{x}}$ defines a rate constant for a respective transition. The values of the rate constants are provided by Supplementary Table 1. (c) Activation and deactivation time courses (gray) of wt channels evoked by ATP jumps at the indicated concentrations. The time courses were globally fitted (blue curves) together with concentration-activation relationship (d) with model F1 shown in b.

new set of rate constants (Supplementary Table 3). Again, all other rate constants were unchanged. The median relative error was also clearly improved compared to that obtained with ${ }^{1}$ global fit of wt ATP data only, but not as good as that obtained with the ${ }^{2}$ global fit for wt ATP and H319K ATP (Fig. 4f). Again, all other rate constants were unchanged. Further improvement of the median relative error was obtained when fitting the wt ATP data set in parallel to the H319K fATP data set by another ${ }^{2}$ global fit, using model F1 and F4 (Supplementary Fig. 4, Supplementary Table 4, Figs. 3d, 4d). Here the factors $f, h, g$ and $j$ were used together accordingly.

To further increase the determinateness of the parameters, it was finally intriguing to increase the constraints by performing a ${ }^{4}$ global fit of all four data sets, i.e. wt ATP, H319K ATP, wt fATP, and H319K fATP (Supplementary Fig. 5) with all four models (Fig. 3). Indeed, the median relative error became smaller again (Fig. 4f). However, the strongly increased constraints in the ${ }^{4}$ global fit produced a directly noticeable mismatch in the equilibrium concentration- $P_{\mathrm{o}}$ relationship of the wt fATP data, predicting less opening than found experimentally (red arrow in Supplementary Fig. 5). We therefore repeated the ${ }^{4}$ global fits with an additional factor to enhance the $P_{\mathrm{o}}$ at higher ligand concentrations. With a single factor, the only possibility turned out to accelerate $k_{7}, k_{9} k_{11}$, i.e. when accelerating the $C_{\mathrm{x}} \rightarrow F_{\mathrm{x}}$ rates. To this end we introduced in model F3 an additional factor $m$ for these parameters and performed the fit de novo, yielding a reasonable description of the data (Fig. $4 \mathrm{a}-\mathrm{e}$ ). The errors of all rate constants were now in an acceptable range below $50 \%$ with the exception of $k_{21}$ which was with $54 \%$ only slightly above (Supplementary Table 6). Accordingly, also the median relative error of the ${ }^{4}$ global fit was lowest (Fig. 4f). We like to emphasize that it is not the increased number of data points which has caused this gain of accuracy because, as described in "Methods", the weight of the data points was artificially kept constant in all fits by appropriate factors.

The ${ }^{4}$ global fit with the coupling factors $f, g, h, j$ and $m$ and the resulting rate constants $k$ for each transition gives us the opportunity for further interpretation of the model. It should be noted that our analysis provided us 
a

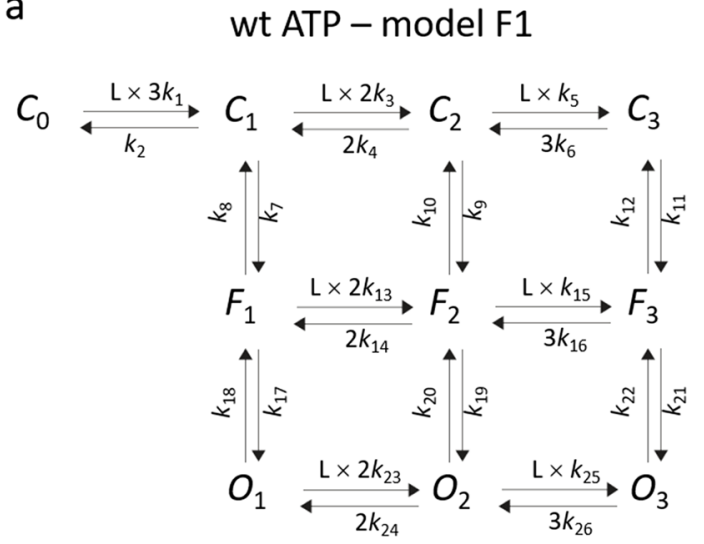

C

\section{wt fATP - model F3}

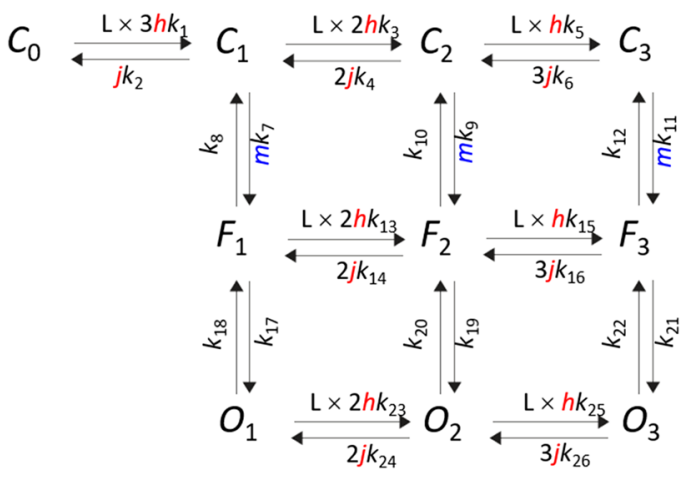

b H319K ATP - model F2

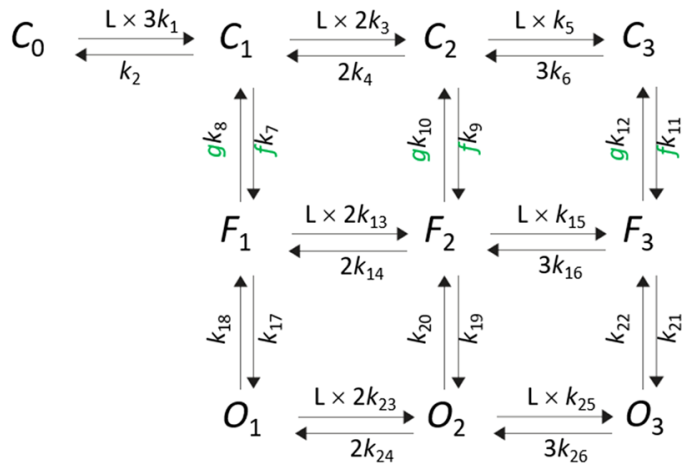

d H319K fATP - model F4

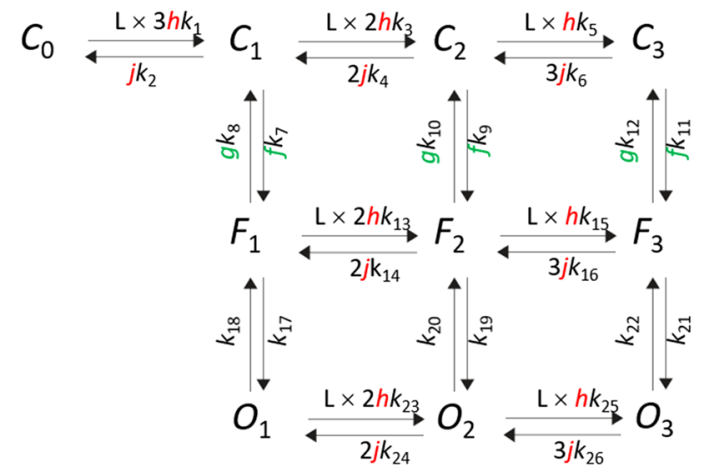

Figure 3. Models used for the extended global fits. In addition to model F1, the models F2, F3 and F4 were used to fit the data of H319K ATP, wt fATP and H319K fATP, respectively. The factors $f, g, h$, and $j$ are coupling factors generating functional differences in the models. $f$ and $g$ were used to accelerate and decelerate the $C_{\mathrm{x}} \rightarrow F_{\mathrm{x}}$ and $F_{\mathrm{x}} \rightarrow C_{\mathrm{x}}$ steps whereas $h C_{\mathrm{x}} \rightarrow F_{\mathrm{x}}$ step of model F3 to enable fitting of the concentration- $P_{\mathrm{o}}$ relationship. For further explanation see text.

three specific factors for isomerisation steps in the model: $f(11.20)$ accelerates the flip step from the closed to the open states in $\mathrm{H} 319 \mathrm{~K}$ whereas $g\left(5.14 \times 10^{-4}\right)$ practically abolishes the respective backward reaction. $m(15.59)$ evokes an accelerated flip in wt channels with fATP, a finding that is to some extent surprising. In contrast, $h$ $(0.17)$ and $j(5.57)$ are modifiers of the binding and unbinding steps, respectively.

Notably, the rate constants $k_{21}\left(F_{3}-O_{3}\right.$ transition $)$ and $k_{22}\left(O_{3}-F_{3}\right.$ transition $)$ in the ${ }^{4}$ global fit are in the range of $10^{11} \mathrm{~s}^{-1}$ which is much faster than the speed limit of our recording system, and they can therefore not be physically interpreted. In an attempt to estimate the influence of these rate constants of the $F_{3}-O_{3}$ step on our conclusions, we computed time courses with the equations used in the ${ }^{4}$ global fit, thereby systematically reducing $k_{21}$ by orders of magnitude while preserving the ratio between $k_{21}$ and $k_{22}$. Using all other determined rate constants, we then evaluated when $\chi^{2}$ increased by more than $1 \%$. This was obtained only when $k_{21}$ was reduced by as much as 8 orders of magnitude to $2.38 \times 10^{3} \mathrm{~s}^{-1}$, resulting in $k_{22}=9.38 \times 10^{2} \mathrm{~s}^{-1}$. These values are well in a physically reasonable range and represent an estimate for a lower border of these transitions. Assuming a speed limit for closed-open transitions of $\sim 10^{6} \mathrm{~s}^{-130}$, all other determined rate constants are slower. It should also be noted, that the rate constants of ligand binding at low concentrations are sufficiently slow to be resolved in our recordings. One of the rate constants of unbinding, $k_{24}$, was much too slow to contribute to a time-dependent change in our measurements. Consequently, this unbinding process did not contribute to the probability fluxes in our probability flux analysis (see below, Fig. 5). The equilibrium constants determined herein seem to be in a realistic, i.e. physically reasonable range.

Occupation of states as function of the ATP concentration at equilibrium. Knowing all constants in model F1 enabled us next to consider the occupancy of all 10 state as function of the ATP concentration in wt channels at equilibrium (Fig. 5a). Thereby, the equilibrium constant of the ultrafast $\mathrm{F}_{3}-\mathrm{O}_{3}$ transition was treated as all other equilibrium constants. At increasing ATP, $C_{0}$ becomes depopulated to progressively fill $C_{1}$ and $C_{2}$ and finally to establish an equilibrium between $F_{3}$ and $O_{3}$. Neither the fully liganded closed state $C_{3}$ nor the partially liganded flipped and open states, $F_{1}, F_{2}, O_{1}$ and $O_{2}$, play a relevant role. Because some of them must be passed for channel opening, they must be instable. In $\mathrm{H} 319 \mathrm{~K}$ channels the situation differs notably (Fig. 5b). Here at increasing ATP $C_{2}$ becomes irrelevant and the double-liganded states $F_{2}$ and $O_{2}$ contribute at intermediate ATP 


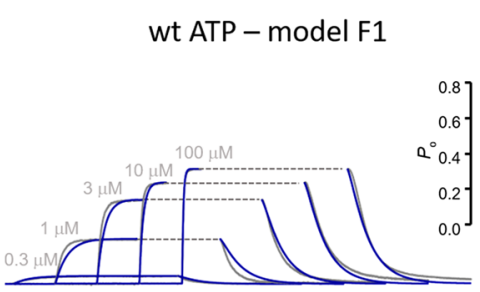

b

H319K ATP - model F2

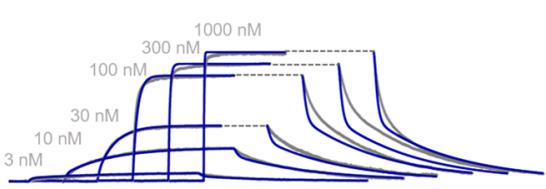

C wt fATP - model F3 d H319K fATP - model F4

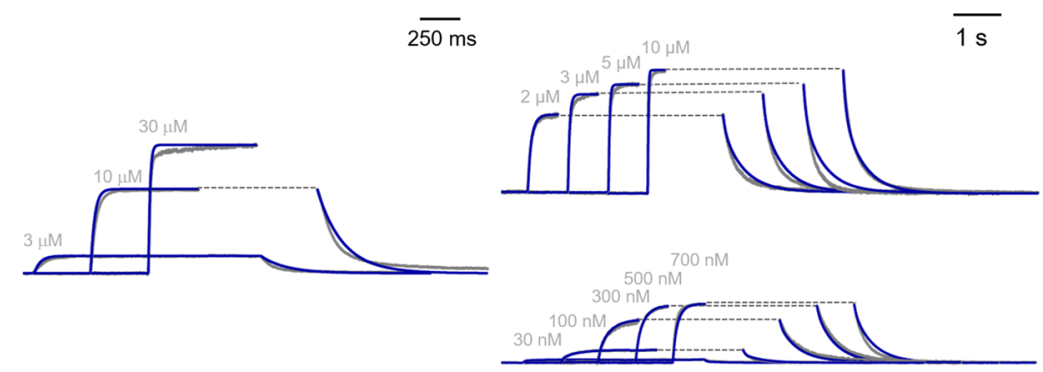

e

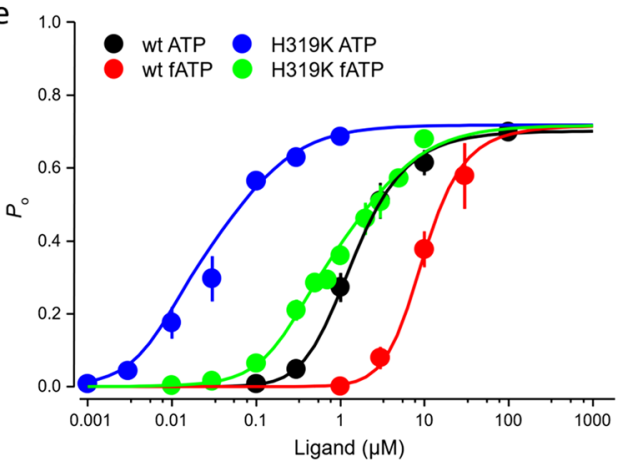

f

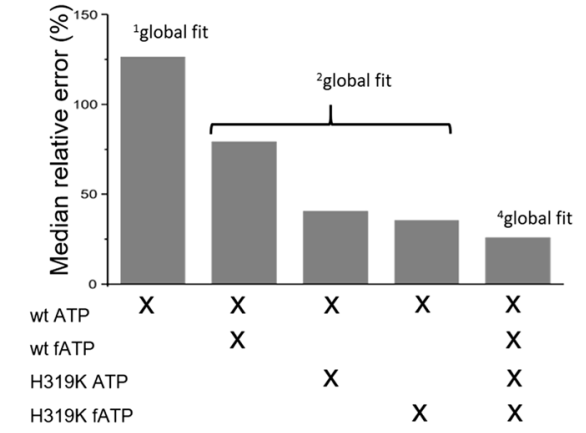

Figure 4. ${ }^{4}$ Global fit. (a-d) Activation and deactivation time courses of the data (gray) wt ATP, H319K ATP, wt fATP, and H319K fATP. The respective ligand concentrations are indicated. (e) Equilibrium concentration$P_{\mathrm{o}}$ relationships corresponding to the concentrations in $(\mathbf{a}-\mathbf{d})$. The data in (a-e) were globally fitted (a-d) blue curves; (e) colored curves) with the models F1, F2, F3 and F4 (Fig. 3) to the data by the ${ }^{4}$ global fit. f Median of the relative error of the parameters obtained by the indicated global fits. The ${ }^{4}$ global fit produced the lowest median of the relative error. For further explanation see text and "Methods".

concentrations of which $\mathrm{O}_{2}$ contributes significantly to $P_{\mathrm{o}}$. At further increasing ATP, again $\mathrm{F}_{3}$ and $\mathrm{O}_{3}$ dominate, similar to wt channels. Hence, populating the double-liganded states $F_{2}$ and $\mathrm{O}_{2}$ leads to the higher apparent affinity of the $\mathrm{H} 319 \mathrm{~K}$ mutant.

Net probability fluxes and transition pathways. Further insights into the channel gating can be gained by considering the probability fluxes in model F1 and F2 and identifying thus the transition pathways of the probability following jumps of the ATP concentration. Using the constants obtained by the ${ }^{4}$ global fit (Supplementary Table 6), we simulated the time courses of the probability $P_{\mathrm{X}}(t)$ that an individual state $\mathrm{X}$ is occupied during the activation and deactivation gating and computed the total net probability fluxes between state $\mathrm{X}$ and $\mathrm{Y}, F_{\mathrm{XY}}$ ( (see "Methods"). This approach allows to identify the transition pathways ${ }^{31-33}$ in the respective model for a given ligand step.

Consider first the net probability fluxes for wt channels after applying $1 \mu \mathrm{M}$ ATP, a concentration near the $E C_{50}$ value (Fig. $5 \mathrm{c}$ ). The predominant pathway to the most relevant open state $\mathrm{O}_{3}$ runs along $C_{1}-C_{2}-F_{2}-F_{3}$, of which $F_{2}$ is less stable than the others (c.f. Figure $5 \mathrm{a}$ ). The pathway along $C_{3}$ instead $F_{2}$ is also used to some extent. When applying the saturating concentration of $100 \mu \mathrm{M}$ ATP (Fig. $5 \mathrm{~d}$ ), the transition pathway runs predominantly through the closed (non-flipped) states and opening is exclusively generated by $\mathrm{O}_{3}$ via $\mathrm{F}_{3}$. A subordinate activation pathway runs along $F_{2}$ instead of $C_{3}$. The transition pathway for deactivation differs from the activation pathway notably. Independent whether activation was partial (Fig. 5c) or complete (Fig. 5d), it runs with high preference through the double-liganded states $\mathrm{O}_{2}-\mathrm{F}_{2}-\mathrm{C}_{2}$ to $\mathrm{C}_{1}$ and $\mathrm{C}_{0}$, generating a pronounced hysteresis in the gating regarding the double- and triple-liganded states.

In $\mathrm{H} 319 \mathrm{~K}$ the net probability fluxes differ noticeably. At $30 \mathrm{nM}$ ATP, again a concentration near the $E C_{50}$ value, the binding of already the first ligand generates a significant flip $C_{1}-F_{1}$, and via $F_{2}$ and $F_{3}$ the relevant open states $\mathrm{O}_{2}$ and $\mathrm{O}_{3}$, respectively, are populated (Fig. 5e). In contrast to wt channels, the saturating concentration of $1 \mu \mathrm{M}$ ATP also favors the pathway $C_{1}-F_{1}-F_{2}-F_{3}$ to generate predominant opening from the triple liganded flipped state $F_{3}$ (Fig. 5f). Notably, also at the same ATP concentration of $1 \mu \mathrm{M}$ the net probability fluxes in wt and $\mathrm{H} 319 \mathrm{~K}$ channels differ upon activation due to the mutation.

Similar to wt channels the predominant transition pathway for deactivation in $\mathrm{H} 319 \mathrm{~K}$ channels differs notably from that of activation, predominantly at the high ATP concentration of $1 \mu \mathrm{M}$, where it runs along $\mathrm{O}_{3}-\mathrm{O}_{2}-\mathrm{F}_{2}$ in contrast to activation which employs $F_{3}$, generating again pronounced hysteresis for the closed-open isomerization. In contrast to wt channels, in $\mathrm{H} 319 \mathrm{~K}$ channels the flipping runs predominantly in both activation and deactivation with a high preference along the pathway $C_{1}-F_{1}-F_{2}$ (Fig. 5 e,f), i.e. without hysteresis (c.f. Figure 5 c,d). 

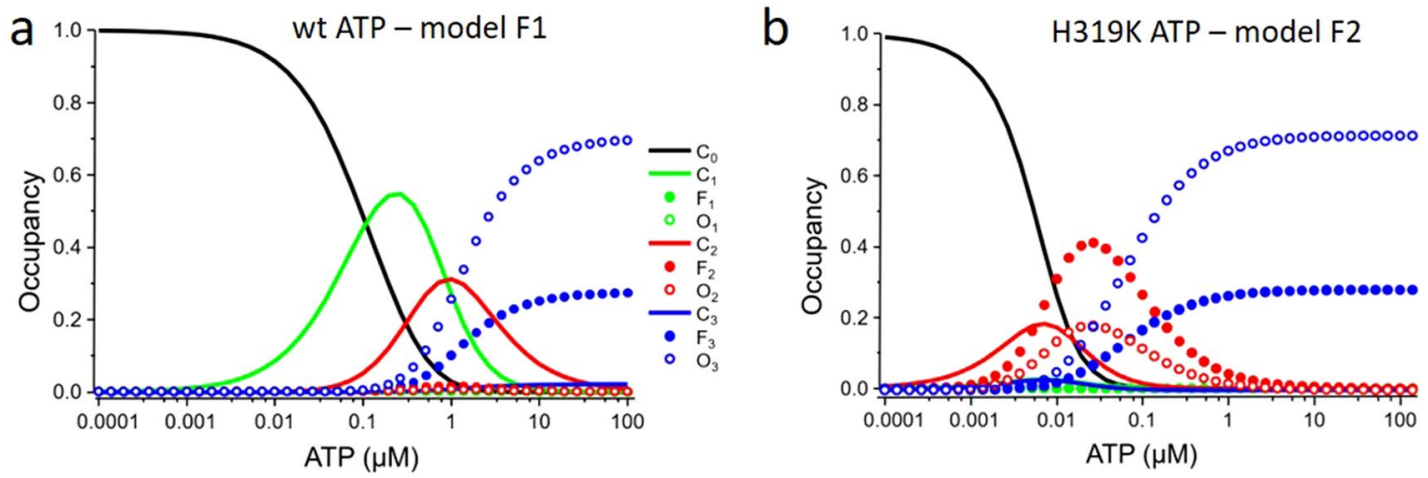

C

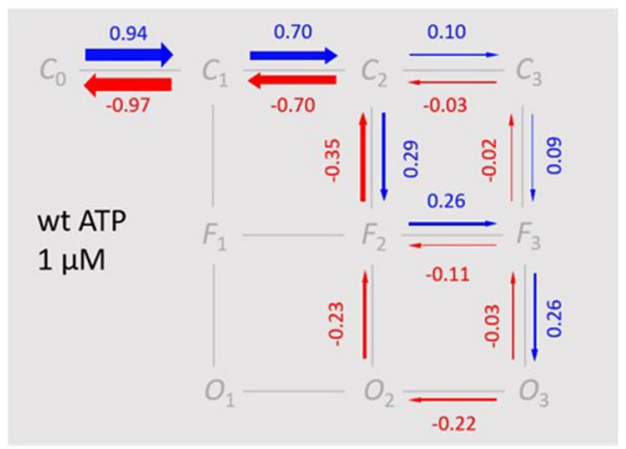

e

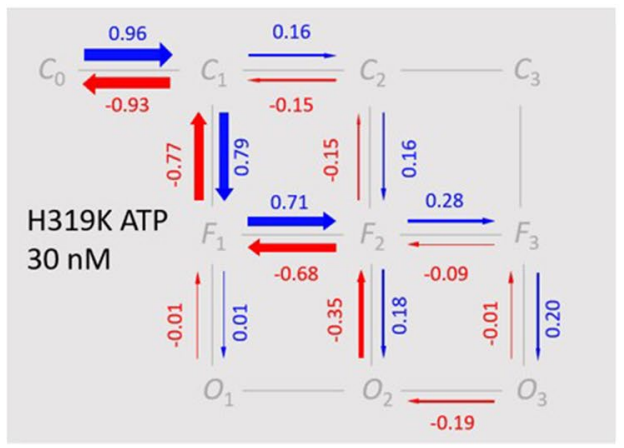

d

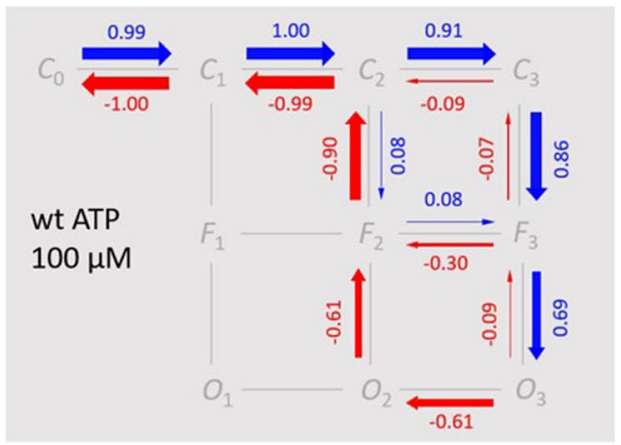

f

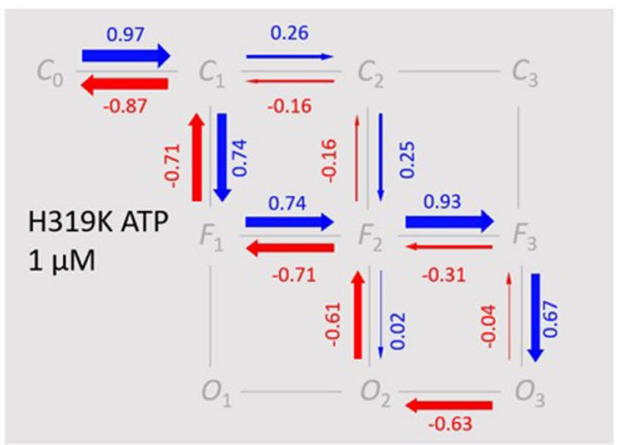

Figure 5. State occupancies at equilibrium and probability fluxes. (a) Occupancy of the states for wt channels as function of the ATP concentration according to model F1. (b) Occupancy of the states for H319K channels as function of the ATP concentration according to model F2. The partially liganded states $\mathrm{F}_{2}$ and $\mathrm{O}_{2}$ are significantly populated and contribute to the higher apparent affinity of the mutant. (c). Net probability fluxes in model F1 for wt channels when jumping the ATP concentration from zero to $1 \mu \mathrm{M}$ (blue arrows) and back to zero (red arrows). The thickness of the arrows symbolizes the amount of net probability flux which is also specified by the adjacent numbers. Net probability fluxes below 0.01 were omitted. (d) As c but with $100 \mu \mathrm{M}$ ATP. $€$ Net probability fluxes in model F2 for H319K channels when jumping the ATP concentration from zero to $30 \mathrm{nM}$ and back to zero. Same symbolism as in (c). (f) As (e) but with $1 \mu \mathrm{M}$ ATP.

Equilibrium free energies and cooperativity. Knowledge of the rate constants, and thus all equilibrium constants, in model F1 allowed us also to estimate the Gibbs free energies for the association in the binding steps, $\Delta G_{\mathrm{A}}$, as well as for the flip steps and closed-open isomerisations, $\Delta G_{\mathrm{E}}$, according to

$$
\Delta G_{A}=-R T \ln \left(L \times k_{x} / k_{x+1}\right)
$$

and

$$
\Delta G_{E}=-R T \ln \left(k_{x} / k_{x+1}\right),
$$

respectively. $R$ is the molar gas constant, $T$ the absolute temperature and $L$ the ligand concentration. Setting $L$ to $1 \mu \mathrm{M}$, a value close to $E C_{50}=1.2 \mu \mathrm{M}$, the $\Delta G_{\mathrm{A}}$ values were attributed to the individual binding steps of model $\mathrm{F} 1$ (blue bars) and the $\Delta G_{\mathrm{E}}$ values to the flip or closed-open isomerisations (ochre bars) (Fig. 6a). There are four major results: (1) The absolute values of $\Delta G_{\mathrm{A}}$ for related steps in the closed, flipped and open states markedly decrease, indicating that flipping and opening successively promote ligand binding. (2) $\Delta G_{\mathrm{A}}$ increases with the degree of liganding for all, closed, flipped and open states, indicating a pronounced negative cooperativity in the binding process. With respect to the second binding step, the degree of $\Delta G_{\mathrm{A}}$ increase for the third binding 
a

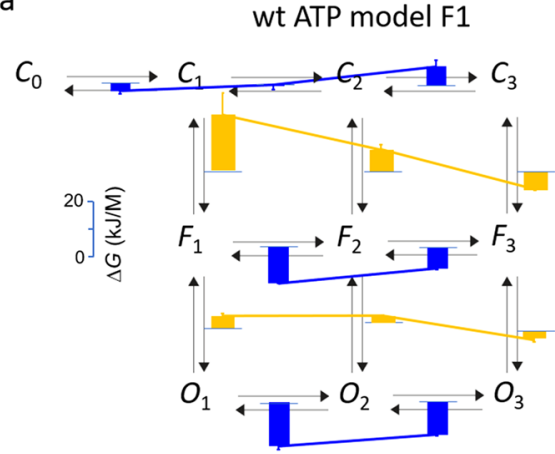

b

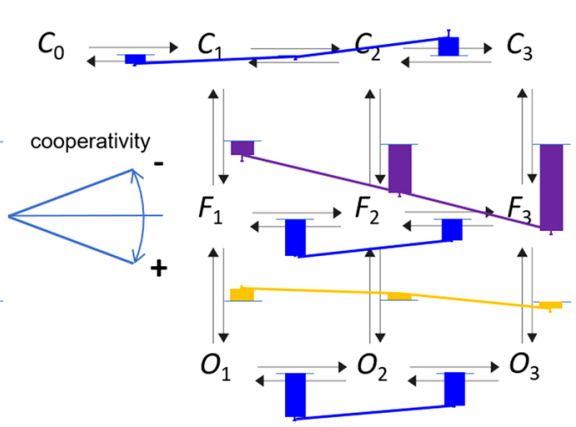

C

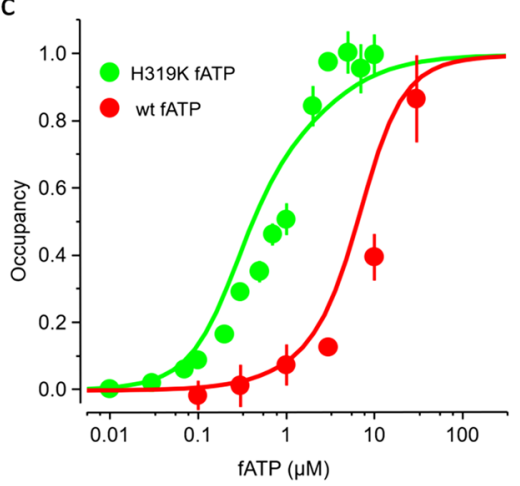

Figure 6. Gibbs free energies and predicted binding. (a) Gibbs free energy in model F1 and F2 for the association in the binding steps, $\Delta G_{\mathrm{A}}$, at the ligand concentration $L=1 \mu \mathrm{M}$ close to the $E C_{50}$ value of $1.2 \mu \mathrm{M}$ (blue bars) and $\Delta G_{\mathrm{E}}$ for the closed-flipped and flipped-open isomerisations (ochre bars), according to model F1. The blue lines indicate negative cooperativity for the binding steps and the ochre lines positive cooperativity for both isomerisations. (b) Same as a but for H319K ATP and with model F2. Compared to wt, $\Delta G_{\mathrm{E}}$ for the closedflipped isomerization in $\mathrm{H} 319 \mathrm{~K}$ is notably decreased at all degrees of liganding, thereby preserving the degree of cooperativity (purple bars and lines). (c) Comparison of experimental fATP binding to wt and H319K channels (data points, $\mathrm{n}=3-6$ ), obtained from a previous study ${ }^{27}$, superimposed by the binding computed by the ${ }^{4}$ global fit (continuous lines).

step is similar for the closed, flipped and open states. Such a negative cooperativity is very surprising because in P2X2 channels concentration-activation relationships are steep and generate typically Hill coefficients around 2 , suggesting positive cooperativity. This cooperativity can therefore only be generated by subsequent reactions. (3) Accordingly, $\Delta G_{\mathrm{E}}$ for the flip reaction strongly decreases with the degree of liganding, necessarily to a higher degree as compared to $\Delta G_{\mathrm{A}}$. Hence, the steeper drop of $\Delta G_{\mathrm{E}}$ for the flip steps at increased liganding, as compared to the corresponding rise of $\Delta G_{\mathrm{A}}$, apparently overcompensates for the negative cooperativity in binding, resulting in an overall positive cooperativity for channel activation. (4) The positive cooperativity in the closed-open isomerisations is much less pronounced than in the flip reactions. Together, these results indicate that it is the flip reaction which introduces the pronounced positive cooperativity in the activation of $\mathrm{P} 2 \mathrm{X} 2$ channels, thereby overbalancing the pronounced negative cooperativity in the binding steps.

When considering the respective process for $\mathrm{H} 319 \mathrm{~K}$ and ATP in terms of our model, the effect of the mutation is solely to lower the energy barriers for the flip reaction, thereby keeping the slope for $\Delta G_{\mathrm{E}}$ (Fig. 6b).

Relating the computed to experimental ligand binding. To further confirm our approach, the concentration dependence of ligand binding has been attributed to the ligand binding determined experimentally by recording the fluorescence intensity of the binding of fATP to P2X 2 channels. The experimental concentration-binding relationships were obtained from a previous report for wt and $\mathrm{H} 319 \mathrm{~K}$ channels ${ }^{27}$ and the theoretical curves obtained from the ${ }^{4}$ global fit (Fig. 4a-e, Supplementary Table 6) were superimposed (Fig. 6c). Despite the fact that the experimental and the predicted relationships were obtained from significantly different conditions, fluorescence-optical analyses in cell cultures versus current recordings with the patch-clamp technique in isolated cells, the relationships match reasonably well.

\section{Discussion}

We performed a detailed kinetic analysis of $\mathrm{P} 2 \mathrm{X} 2$ receptors that was based on equilibrium and non-equilibrium data at multiple ligand concentrations, extended by data from the mutant $\mathrm{H} 319 \mathrm{~K}$, positioned in the middle of the transmission pathway, and data obtained with the fluorescent ligand of reduced apparent affinity ${ }^{27}$. We investigated the resulting four data sets with four intimately coupled kinetic schemes (model F1-F4). These results allowed us to identify a surprising, but also simple, cooperativity pattern for wild type P2X2 channels and to recognize that the typically steep concentration- $P_{\mathrm{o}}$ relationship for these channels is the result of a positively cooperative flip reaction, overbalancing a negative cooperativity in ATP binding. Moreover, we identified a marked hysteresis of the net probability fluxes in an activation-deactivation cycle for the third binding step and that the facilitated opening in the H319K mutant is caused by a significantly enhanced flipping with only one ligand bound.

The result of coexisting negative cooperativity in the binding and positive cooperativity in the flip step shows that in P2X2 channels the subunits essentially interact already at the initial stages of activation and not only in the concerted closed-open reaction of the pore as suggested earlier ${ }^{11}$. This initial interaction is present in both the binding process itself and the transmission of the signal along the $\beta-14$ sheet as part of the conformational flip. Moreover, our results show that in wild type P2X2 channels relevant activation requires all three subunits, confirming on the one hand previous reports ${ }^{10,13-15}$, but conflicting on the other with reports suggesting that two subunits suffice for full activation ${ }^{10,11}$. The existence of an intermediate flip step in the activation pathway has already been suggested by three other studies using markedly different experimental approaches, for P2X2 channels with either a kinetic analyses ${ }^{34}$ or by using covalently bound 8 -thiocyano-ATP ${ }^{35}$, as well as for P2X7 
and $\mathrm{P} 2 \mathrm{X} 2$ channels with photo-affinity labeling using 2(3)-O-(4-benzoylbenzoyl)-ATP ${ }^{36}$. It remains to be shown to what extent the flip characterized herein corresponds to the flip reported in these studies. Our study did not yield the necessity to include five discernable key steps in the activation process ${ }^{18}$. Usage of a model with three binding steps for trimeric channels is certainly plausible for a trimeric channel. Therein, however, the combination of pronounced negative cooperativity for ligand binding, i.e. the binding of a ligand inhibits the binding of the next ligand, and even more pronounced positive cooperativity for the flip reaction, i.e. the binding of the next ligand facilitates the flip more than the binding of the previous ligand, is a new molecular mechanism of cooperative activation gating in P2X channels. An increased binding affinity for intermediate flipped states compared to closed states has been shown previously for nicotinic acetylcholine $\mathrm{e}^{29}$ and glycine receptors ${ }^{37}$.

The major methodological impact of our approach is that extended global fits with complex and intimately coupled kinetic schemes can be effectively used to study multiple data and that this approach enabled us to identify as much as 26 rate constants in model F1, topping all our previous approaches in CNG and HCN channels, even when using orthogonal binding data in addition to current data ${ }^{24-26}$. For CNGA2 channels this led to the identification of a cooperativity sequence in the cGMP binding of 'negative-positive-no' for the second, third and fourth binding step ${ }^{24}$ whereas for HCN2 channels this corresponding sequence was 'positive-negative-positive' for channels pre-activated by hyperpolarizing voltage ${ }^{26}$ and 'negative-no-positive' at depolarized voltages ${ }^{38}$. Based on global fits of ligand-induced activation and deactivation solely, as performed herein, we already previously showed for CNGA2 channels intricate cooperativity ${ }^{22}$, as we did by analyzing voltage-induced activation and deactivation time courses of tetrameric HCN2 channels ${ }^{23}$. Global fits provided also essentially new insights into the function of other ion channels, e.g. for the gating charge movement in Shaker channels ${ }^{21}$ and for the effects of permeating $\mathrm{K}^{+}$ions on the $I V$-relationships of viral Kv channels ${ }^{20}$, all measured in ensemble currents and under equilibrium conditions. Also single-channel currents were subjected to global fit approaches, as shown elegantly e.g. for glycine receptors ${ }^{39}$. Furthermore, our extended global fit approach is promising to gain more insights in other P2X receptor subtypes for instance the enigmatic process of pore dilatation in P2X7 receptors ${ }^{40,41}$ or in the function of individual subunits in heteromeric assemblies ${ }^{42}$.

To some extent our extended global fit analysis is related to the widely used double-mutant cycle analysis ${ }^{43}$, determining Gibbs free energy differences for two single and the corresponding double mutant to specify free energies of interaction ${ }^{44}$. An often used approach for analyzing ion channels in macroscopic recordings is to determine $E C_{50}$ values and translate them to free energies of interaction ${ }^{45}$. Compared to these analyses, our approach includes more extensive functional information from the channel than simply using $E C_{50}$ values (1) by fitting activation and deactivation time courses in their concentration-dependence and also the steepness of the $P_{\mathrm{o}}$ relationship, (2) by using the combination of a mutation and a second ligand with different apparent affinity, and (3) by fitting a system of four intimately coupled kinetic schemes with three ligand binding sites which is physically much more meaningful compared to a simple $E C_{50}$ value. Global fits were also used previously to thermodynamically characterize the function and interaction of proteins ${ }^{46}$, thereby modulating the proteins by mutations ${ }^{4-49}$ or by molecules functionally interacting with the proteins ${ }^{50-52}$. Another strategy is to globally fit orthogonal observables, such as e.g. calorimetric, spectroscopic or additional biosensing experiment $5^{53}$.

In conclusion, we provide a plausible hypothesis for the steep concentration- $P_{o}$ relationship resulting from a negatively cooperative ATP binding countered by occupancy-dependent intermediate flip transitions. These results shed light into the conformational changes proceeding in the activation of $\mathrm{P} 2 \mathrm{X}$ receptors.

\section{Materials and methods}

Synthesis of fATP. The synthesis of the novel fluorescent ATP derivative 2-[DY-547P1]-AET-ATP (fATP) has been described previously ${ }^{27}$.

Molecular biology and cell culture. Rat wt P2X2 (RRID: Addgene_137071) and mutant P2X2 H319K (RRID: Addgene_137072) in pcDNA5/FRT/TO and derived stable cell lines (RRID: AC line CVCL_YJ33 for P2X2 wt, RRID: AC line CVCL_YJ34 for P2X2H319K) has been described previously (Sattler et al.). HEK293 cell lines containing an inducible promoter (Flp-In-T-REx 293, Invitrogen) were cultured in MEM supplemented with $10 \%$ FCS, non-essential amino acids (Gibco) and antibiotics according to the instructions of the manufacturers. Cell lines were used up to a passage number of 20. For most macroscopic measurements, cells were seeded on glass coverslips and used $24-48 \mathrm{~h}$ after tetracycline induction. For single-channel and macroscopic current recordings subjected to noise analysis, cells were used 2-6 h after induction to keep expression reasonably low.

Electrophysiology. Recording of macroscopic currents was performed with a standard patch-clamp technique in the whole-cell mode ${ }^{54}$. The patch pipettes were pulled from borosilicate glass (ID $1.0 \mathrm{~mm}$, OD $2.0 \mathrm{~mm}$; Hilgenberg, Malsfeld, Germany) using a micropipette puller (P-97, Sutter Instrument, Novato, USA). The pipettes were filled with intracellular solution containing (mM) $142 \mathrm{NaCl}, 5 \mathrm{BAPTA}, 5$ EGTA and $10 \mathrm{HEPES}, \mathrm{pH}$ 7.4. The pipette resistance was 2.5-6.0 M . The bath solution contained (mM) $142 \mathrm{NaCl}, 10 \mathrm{EGTA}, 10$ HEPES and 10 Glucose, $\mathrm{pH}$ 7.4. For current recording the cells were lifted from the chamber bottom by the patch pipette and positioned in front of the outlet of the application system.

Solution switches were carried out with a theta-glass pipette (inner diameter $\sim 100 \mu \mathrm{m}, \mathrm{WPI}$ ), mounted on a piezo device, or with a three-barrel glass (inner diameter $\sim 600 \mu \mathrm{m}$, Warner Instruments), controlled by a step motor (SF-77B, Warner Instruments). The speed of the laminar solution flow out of the barrels was $2-5 \mathrm{~cm} / \mathrm{s}$. The time of the switch around a cell at the patch pipette was determined to be below $10 \mathrm{~ms}$ by switching between different salt solutions. This time was sufficiently short with respect to the current time courses. Saturation of activation was determined with ATP at $100 \mu \mathrm{M}$ for wt P2X2 and $1 \mu \mathrm{M}$ for P2X2 H319K. The currents were 
recorded with an Axopatch 200B or HEKA EPC 10 amplifier in combination with the ISO3 or Patchmaster and software. The sampling rate was either 2 or $10 \mathrm{kHz}$ and the recordings were on-line filtered at 1 and $2.9 \mathrm{kHz}$, respectively, using a 4-pole Bessel filter. Recordings for noise analysis were sampled at $50 \mathrm{kHz}$ and filtered at $10 \mathrm{kHz}$. The currents were recorded at a constant holding potential of $-50 \mathrm{mV}$.

Single-channel recordings were performed in the cell-attached configuration at $-50 \mathrm{mV}$. The pipettes were pulled from quartz tubing (ID $0.7 \mathrm{~mm}$, OD $1.0 \mathrm{~mm}$; Science Products, Hofheim, Germany) using a micropipette puller (P-2000, Sutter Instruments, Novato; USA). Pipettes were filled with intracellular solution (mM $142 \mathrm{NaCl}$, 5 BAPTA, 5 EGTA and 10 HEPES, pH 7.4) and an ATP concentration near the $E C_{50}$ value $(1 \mu \mathrm{M}$ wt, $30 \mathrm{nM}$ $\mathrm{H} 319 \mathrm{~K})$. The pipette resistance was $10-15 \mathrm{M} \Omega$. The resting potential of the cells was zeroed by the potassium chloride bath solution (mM $142 \mathrm{KCl}, 10 \mathrm{EGTA}, 10 \mathrm{HEPES}, 10$ glucose, $\mathrm{pH} 7.4$ with $\mathrm{KOH}$ ). Data were sampled at $20 \mathrm{kHz}$ and on-line filtered at $5 \mathrm{kHz}$ using a 4-pole Bessel filter.

Conventional data evaluation. Concentration-activation relationships were constructed from the maximum currents during a solution application. These current amplitudes were normalized with respect to the current at saturating ATP (wt $100 \mu \mathrm{M} ; \mathrm{H} 319 \mathrm{~K} 1 \mu \mathrm{M}$ in each individual cell). In Fig. 1a the resulting data points were fitted with

$$
I / I_{\max }=1 /\left(1+\left(E C_{50} /[\mathrm{X}]\right)^{H}\right)
$$

with the Origin 8.5.1 software using a non-linear curve fitting routine with statistical weighting. $I$ is the actual current amplitude and $I_{\max }$ the maximum current amplitude at saturating ATP. $E C_{50}$ is the ligand concentration generating half maximum current. $H$ is the respective Hill coefficient. [X] is the actual ligand concentration of ATP.

The amplitude of the single-channel current was calculated from data filtered digitally down to $1 \mathrm{kHz}$ and respective amplitude histograms were fitted with the sum of $j=2$ Gaussian functions (Fig. 1b).

$$
y=\sum_{j=1}^{2} A_{j} \exp \left(-\frac{\left(i-i_{j}\right)^{2}}{2 \sigma_{j}^{2}}\right)
$$

$A_{j}$ is a calibration factor, $i$ the amplitude of the actual single-channel current and $i_{j}$ the mean amplitude of the single-channel current for the closed or the open channel whose difference provides the mean single-channel current $i_{s}$.

Experimental data are given as mean \pm s.e.m.

Global fit strategies. Each applied concentration pulse protocol consisted of a step to a defined concentration and, after a specified time, of a step back to zero concentration to evoke activation and deactivation time courses, respectively. The activation time courses from switching on the ligand concentration until the maximum and the deactivation time courses were analyzed. All time-dependent currents at the different concentrations were normalized to the respective current maximum to achieve in the fit for each current the same weight, even if the amplitude differed by orders of magnitude. These time courses were fitted together with steadystate activation relationships. Systems of first-order differential equations were used. Each differential equation describes the time-dependent change of the occupational probability $d p_{\mathrm{x}} / d t$ of a considered state $\mathrm{x}$ resulting from the sum of all inputs and outputs.

This results in a system of $n$ differential equations $d p_{\mathrm{x}} / d t(\mathrm{x}=1 \ldots n) . \mathrm{x}$ denotes the $\mathrm{n}$ states of the model. Consider for example model F1 (Fig. 2b) with x running from $\mathrm{C}_{0}$ to $\mathrm{O}_{3}$ :

$$
\begin{gathered}
d p_{\mathrm{C} 0} / d t=k_{2} \times p_{\mathrm{C} 1}-L \times 3 k_{1} \times p_{\mathrm{C} 0} \\
d p_{\mathrm{C} 1} / d t=L \times 3 k_{1} \times p_{\mathrm{C} 0}+2 k_{4} \times p_{\mathrm{C} 2}+k_{8} \times p_{\mathrm{F} 1}-\left(k_{2}+L \times 2 k_{3}+k_{7}\right) \times p_{\mathrm{C} 1} \\
\cdot \\
\cdot \\
\cdot \\
d p_{\mathrm{O} 3} / d t=L \times k_{25} \times p_{\mathrm{O} 2}+k_{21} \times p_{\mathrm{F} 3}-\left(3 k_{26}+k_{22}\right) \times p_{\mathrm{O} 3}
\end{gathered}
$$

$L$ is the ligand concentration and $k_{\mathrm{a}}$ denotes a rate constant.

The system of differential Eqs. (6) can be written in a compact form in matrix notation as follows:

$$
\frac{d p}{d t}=\operatorname{coef}(L) \cdot p(t)
$$

$p(t)$ is the column vector of the probabilities to be in one of the states $\mathrm{C}_{0}$ to $\mathrm{O}_{3}$.

$\operatorname{coef}(L)$ is the matrix of the coefficients of the differential equation system depending on the concentration $L$. $\frac{d p}{d t}$ is the column vector of the derivatives of the probabilities.

An own program was developed with the Matlab software to fit the data. Because we didn't use any constraints concerning the rate constants, the number of fit parameters was equal to the number of rate constants.

The system of differential Eqs. $(6,7)$ was solved numerically using the Eigenvalue method. As a result, we got for each fit point of the experimental curves numerical values of the occupation of states. The sum of the 
occupation of all open states is the open probability. In this way, the $P_{\mathrm{o}}$ vs. time courses and the steady-state $P_{\mathrm{o}}$ vs. concentration curves were calculated from the set of model parameters. Varying the model parameters step by step using a modified Levenberg-Marquardt algorithm ${ }^{53}$ the program finds an optimal set of parameters for a minimum difference between the experimental and the calculated curves.

The criterion for the best fit was the minimized sum of the squared deviations $S$ according to

$$
S=\sum_{i=1}^{n_{t}} u \sum_{j=1}^{n_{d}} \frac{\left(I_{m}\left(t_{i j}\right)-I_{c}\left(t_{i j}\right)\right)^{2}}{\sigma_{I}^{2}\left(t_{i j}\right)}+\sum_{k=1}^{n_{s}} s_{k} \sum_{v=1}^{n_{x}} \frac{\left(P_{o m}\left(x_{k v}\right)-P_{o c}\left(x_{k v}\right)\right)^{2}}{\sigma_{P o}^{2}\left(x_{k v}\right)}
$$

The fitting process was terminated when the relative change of $S$ in Eq. (8) from one iteration step to the next was below a set border.

$I_{\mathrm{m}}\left(t_{i j}\right)$ and $I_{\mathrm{c}}\left(t_{i j}\right)$ are the normalized measured and calculated current amplitudes of a fit point inside a trace $i$ at a time $j . n_{\mathrm{t}}$ denotes the number of time traces, $n_{\mathrm{d}}$ the number of fit points in a trace, $n_{\mathrm{s}}$ the number of steadystate activation relationships used, and $n_{\mathrm{x}}$ the number of concentrations in a particular steady-state activation relationship. $P_{o m}\left(x_{k v}\right)$ and $P_{o c}\left(x_{k v}\right)$ are the steady-state values of the measured and calculated open probabilities at a concentration $v$ in a steady-state activation relationship $k . \sigma_{I}^{2}\left(t_{i j}\right)$ is the measured variance at time $j$ of a fit point in a particular trace $i\left(i=1 \ldots n_{t}\right)$ and $\sigma_{\mathrm{Po}}^{2}\left(x_{k v}\right)$ is the measured variance at concentration $v$ in a particular steady-state activation relationship $k$. The squared deviations of the currents and the steady-state values in Eq. (8) were weighted by the reciprocal variances $\sigma_{I}^{2}\left(t_{i j}\right)$ and $\sigma_{\mathrm{Po}_{\mathrm{o}}}^{2}\left(x_{k v}\right)$ of the fit points to assign the better determined fit points a higher weight. The variances $\sigma_{I}^{2}\left(t_{i j}\right)$ and $\sigma_{\mathrm{Po}}^{2}\left(x_{k v}\right)$ for each fit point were experimentally determined.

The weight of each curve in the fit process depends on the used number of fit points.

In the current time courses, the number of fit points was much bigger than that in the concentration- $P_{\mathrm{o}}$ relationships. Therefore, we employed in Eq. (8) the weighting factors $u$ and $s_{k}$ to give all time courses the same weight in the fit as all concentration- $P_{\mathrm{o}}$ relationships. By introducing these factors we achieved for all fits comparability of the errors of the fit parameters. The factors $u$ and $s_{k}$ were calculated such that the effective number of fit points of all current time courses equaled that of the concentration- $P_{\mathrm{o}}$ relationships. The effective number of fit points was obtained by the product of the number of fit points and the weighting factor. For a particular fit, $u$ has the same value for all current time courses. $s_{k}$ is a special factor for each particular concentration- $P_{\mathrm{o}}$ relationship correcting for the different number of fit points in the individual concentration- $P_{\mathrm{o}}$ relationships.

Hence, in all global fits we used 470 effective fit points for all time courses and 470 effective fit points for all concentration- $P_{\mathrm{o}}$ relationships.

As an example, the ${ }^{4}$ global fit employed $n_{t}=47$ time traces and $n_{s}=4$ steady-state relationships. We used for each time trace 100 fit points and for the steady-state relationships all experimentally determined data points (6, wt ATP; 4, wt fATP; 7, H319K ATP; 11, H319K fATP). Assigning each time trace the weighting factor $\mathrm{u}=0.1$ and the steady-state relationships the resulting weighting factors $s_{\mathrm{k}}=19.59,29.38,16.79$ and 10.68, respectively, we obtained the same number of 470 effective fit points for the time traces and for the steady-state relationships. This approach allowed us to directly compare the error of the resulting parameters of the fits even though they use different numbers of data points and data sets.

With the help of the $i$-th entry of the covariance matrix main diagonal $\left(\operatorname{cov}_{i i}\right)$ the standard error for the $i$-th parameter $s e\left(p_{i}\right)$ was calculated as

$$
s e\left(p_{i}\right)=\sqrt{\operatorname{cov} i i}
$$

Probability flux densities and transition pathway analysis. Using the constants obtained by the ${ }^{4}$ global fit with models 3, 3b, 3c and 3d (Supplementary Table 6), the time courses of the occupational probability $p_{\mathrm{X}}(t)$ of the individual states $\mathrm{X}$ were computed for both activation and deactivation. From these time courses the unidirectional probability flux density from state $\mathrm{X}$ to an adjacent state $\mathrm{Y}$ was calculated by

$$
f_{\mathrm{u}, \mathrm{XY}}(t)=p_{\mathrm{X}}(t) \times k_{\mathrm{XY}},
$$

where $k_{\mathrm{XY}}$ is the rate constant specifying the transition from state $\mathrm{X}$ to state $\mathrm{Y}$. The net probability flux density between these two adjacent states was obtained from the difference between the forward and backward unidirectional flux density according to

$$
f_{\mathrm{XY}}=f_{\mathrm{u}, \mathrm{XY}}-f_{\mathrm{u}, \mathrm{YX}}
$$

In model 3, a flux in the direction to $\mathrm{O}_{3}$ was assigned a positive sign. From the net probability flux densities, $f_{\mathrm{XY}}$, the total net probability fluxes, $F_{\mathrm{XY}}$, can be computed by the time integral over the time interval from the concentration jump $(t=0 \mathrm{~s})$ to an end time, $t_{\text {end }}$,

$$
F_{\mathrm{XY}}=\int_{t=0}^{t_{\mathrm{end}}} f_{\mathrm{XY}} d t
$$

For both, activation and deactivation, $t_{\text {end }}$ was set to the end of the time courses used for fitting. As mentioned above we used for the fit process the activation curves from the beginning of the concentration jump until the maximum of the curve to ignore slow desensitization components. For the deactivation curves after removal of ATP, $t_{\text {end }}$ was $5000 \mathrm{~ms}$ or $8000 \mathrm{~ms}$. The resulting total net probability fluxes during activation and deactivation for the four data sets are shown in Fig. 5c-f. 
Received: 16 July 2020; Accepted: 25 November 2020

Published online: 10 December 2020

\section{References}

1. Hausmann, R., Kless, A. \& Schmalzing, G. Key sites for P2X receptor function and multimerization: overview of mutagenesis studies on a structural basis. Curr. Med. Chem. 22, 799-818 (2015).

2. Khakh, B. S. \& North, R. A. Neuromodulation by extracellular ATP and P2X receptors in the CNS. Neuron 76, 51-69 (2012)

3. Egan, T. M., Samways, D. S. \& Li, Z. Biophysics of P2X receptors. Pflugers Arch. 452, 501-512 (2006).

4. Coddou, C., Yan, Z., Obsil, T., Huidobro-Toro, J. P. \& Stojilkovic, S. S. Activation and regulation of purinergic P2X receptor channels. Pharmacol. Rev. 63, 641-683 (2011).

5. Surprenant, A. \& North, R. A. Signaling at purinergic P2X receptors. Annu. Rev. Physiol. 71, 333-359 (2009).

6. Hattori, M. \& Gouaux, E. Molecular mechanism of ATP binding and ion channel activation in P2X receptors. Nature 485, 207-212 (2012).

7. Kawate, T., Michel, J. C., Birdsong, W. T. \& Gouaux, E. Crystal structure of the ATP-gated P2X(4) ion channel in the closed state. Nature 460, 592-598 (2009).

8. Mansoor, S. E. et al. X-ray structures define human P2X(3) receptor gating cycle and antagonist action. Nature 538, 66-71 (2016).

9. Li, M., Chang, T. H., Silberberg, S. D. \& Swartz, K. J. Gating the pore of P2X receptor channels. Nat. Neurosci. 11, 883-887 (2008).

10. Stelmashenko, O. et al. Activation of trimeric P2X2 receptors by fewer than three ATP molecules. Mol. Pharmacol. 82, 760-766 (2012).

11. Keceli, B. \& Kubo, Y. Signal transmission within the P2X2 trimeric receptor. J. Gen. Physiol. 143, 761-782 (2014).

12. Hausmann, R. et al. ATP binding site mutagenesis reveals different subunit stoichiometry of functional P2X2/3 and $\mathrm{P} 2 \mathrm{X} 2 / 6$ receptors. J. Biol. Chem. 287, 13930-13943 (2012).

13. Fryatt, A. G., Dayl, S., Cullis, P. M., Schmid, R. \& Evans, R. J. Mechanistic insights from resolving ligand-dependent kinetics of conformational changes at ATP-gated P2X1R ion channels. Sci. Rep. 6, 32918 (2016).

14. Ding, S. \& Sachs, F. Single channel properties of P2X2 purinoceptors. J. Gen. Physiol. 113, 695-720 (1999).

15. Jiang, L. H. et al. Subunit arrangement in P2X receptors. J. Neurosci. 23, 8903-8910 (2003).

16. Du, J., Dong, H. \& Zhou, H. X. Gating mechanism of a P2X4 receptor developed from normal mode analysis and molecular dynamics simulations. Proc. Natl. Acad. Sci. U.S.A. 109, 4140-4145 (2012).

17. Browne, L. E. et al. P2X receptor channels show threefold symmetry in ionic charge selectivity and unitary conductance. Nat. Neurosci. 14, 17-18 (2011)

18. Habermacher, C., Dunning, K., Chataigneau, T. \& Grutter, T. Molecular structure and function of P2X receptors. Neuropharmacology 104, 18-30 (2016).

19. Wongsamitkul, N. et al. Quantifying the cooperative subunit action in a multimeric membrane receptor. Sci. Rep. 6, 20974 (2016).

20. Rauh, O., Hansen, U. P., Scheub, D. D., Thiel, G. \& Schroeder, I. Site-specific ion occupation in the selectivity filter causes voltagedependent gating in a viral K(+) channel. Sci. Rep. 8, 10406 (2018).

21. Lacroix, J. J., Hyde, H. C., Campos, F. V. \& Bezanilla, F. Moving gating charges through the gating pore in a Kv channel voltage sensor. Proc. Natl. Acad. Sci. U.S.A. 111, E1950-1959 (2014).

22. Nache, V. et al. Activation of olfactory-type cyclic nucleotide-gated channels is highly cooperative. J. Physiol. 569, 91-102 (2005).

23. Hummert, S. et al. Activation gating in HCN2 channels. PLoS Comput. Biol 14, e1006045 (2018).

24. Biskup, C. et al. Relating ligand binding to activation gating in CNGA2 channels. Nature 446, 440-443 (2007).

25. Nache, V., Eick, T., Schulz, E., Schmauder, R. \& Benndorf, K. Hysteresis of ligand binding in CNGA2 ion channels. Nat. Commun. 4, 2866 (2013).

26. Kusch, J. et al. How subunits cooperate in cAMP-induced activation of homotetrameric HCN2 channels. Nat. Chem. Biol. 8, 162-169 (2012).

27. Sattler, C. et al. Relating ligand binding to activation gating in P2X2 receptors using a novel fluorescent ATP derivative. J. Neurochem. 154(3), 251-262 (2020).

28. Clyne, J. D., LaPointe, L. D. \& Hume, R. I. The role of histidine residues in modulation of the rat P2X(2) purinoceptor by zinc and pH. J Physiol 539, 347-359 (2002).

29. Mukhtasimova, N., Lee, W. Y., Wang, H. L. \& Sine, S. M. Detection and trapping of intermediate states priming nicotinic receptor channel opening. Nature 459, 451-454 (2009).

30. Chakrapani, S. \& Auerbach, A. A speed limit for conformational change of an allosteric membrane protein. Proc. Natl. Acad. Sci. U.S.A. 102, 87-92 (2005).

31. Prinz, J. H., Keller, B. \& Noe, F. Probing molecular kinetics with Markov models: metastable states, transition pathways and spectroscopic observables. Phys. Chem. Chem. Phys. 13, 16912-16927 (2011).

32. Noe, F., Schutte, C., Vanden-Eijnden, E., Reich, L. \& Weikl, T. R. Constructing the equilibrium ensemble of folding pathways from short off-equilibrium simulations. Proc. Natl. Acad. Sci. U.S.A. 106, 19011-19016 (2009).

33. Benndorf, K., Kusch, J. \& Schulz, E. Probability fluxes and transition paths in a Markovian model describing complex subunit cooperativity in HCN2 channels. PLoS Comput. Biol. 8, e1002721 (2012).

34. Moffatt, L. \& Hume, R. I. Responses of rat P2X2 receptors to ultrashort pulses of ATP provide insights into ATP binding and channel gating. J. Gen. Physiol. 130, 183-201 (2007).

35. Jiang, R. et al. Intermediate closed channel state(s) precede(s) activation in the ATP-gated P2X2 receptor. Channels (Austin) 6, 398-402 (2012).

36. Browne, L. E. \& North, R. A. P2X receptor intermediate activation states have altered nucleotide selectivity. J. Neurosci. 33, 14801-14808 (2013).

37. Lape, R., Colquhoun, D. \& Sivilotti, L. G. On the nature of partial agonism in the nicotinic receptor superfamily. Nature 454, 722-727 (2008)

38. Thon, S., Schulz, E., Kusch, J. \& Benndorf, K. Conformational Flip of nonactivated HCN2 channel subunits evoked by cyclic nucleotides. Biophys. J. 109, 2268-2276 (2015).

39. Burzomato, V., Beato, M., Groot-Kormelink, P. J., Colquhoun, D. \& Sivilotti, L. G. Single-channel behavior of heteromeric alpha1beta glycine receptors: an attempt to detect a conformational change before the channel opens. J. Neurosci. 24, 10924-10940 (2004).

40. Virginio, C., MacKenzie, A., Rassendren, F. A., North, R. A. \& Surprenant, A. Pore dilation of neuronal P2X receptor channels. Nat. Neurosci. 2, 315-321 (1999).

41. Li, M., Toombes, G. E., Silberberg, S. D. \& Swartz, K. J. Physical basis of apparent pore dilation of ATP-activated P2X receptor channels. Nat. Neurosci. 18, 1577-1583 (2015).

42. Kowalski, M. et al. Flexible subunit stoichiometry of functional human P2X2/3 heteromeric receptors. Neuropharmacology 99 , 115-130 (2015).

43. Carter, P. J., Winter, G., Wilkinson, A. J. \& Fersht, A. R. The use of double mutants to detect structural changes in the active site of the tyrosyl-tRNA synthetase (Bacillus stearothermophilus). Cell 38, 835-840 (1984)

44. Kusch, J. et al. Role of the S4-S5 linker in CNG channel activation. Biophys J 99, 2488-2496 (2010). 
45. Hidalgo, P. \& MacKinnon, R. Revealing the Architecture of a K+ channel pore through mutant cycles with a peptide inhibitor. Science 268, 307-310 (1995).

46. Beechem, J. M. Global analysis of biochemical and biophysical data. Methods Enzymol. 210, 37-54 (1992),

47. Ionescu, R. M. \& Eftink, M. R. Global analysis of the acid-induced and urea-induced unfolding of staphylococcal nuclease and two of its variants. Biochemistry 36, 1129-1140 (1997).

48. Zhang, M., Case, D. A. \& Peng, J. W. Propagated perturbations from a peripheral mutation show interactions supporting WW domain thermostability. Structure 26, 1474-1485 (2018).

49. Mann, D. et al. Mechanism of the intrinsic arginine finger in heterotrimeric G proteins. Proc. Natl. Acad. Sci. U.S.A. 113, E8041E8050 (2016).

50. Blevins, S. J. \& Baker, B. M. Using global analysis to extend the accuracy and precision of binding measurements with $\mathrm{T}$ cell receptors and their peptide/MHC ligands. Front. Mol. Biosci. 4, 2 (2017).

51. Jamakhandi, A. P., Kuzmic, P., Sanders, D. E. \& Miller, G. P. Global analysis of protein-protein interactions reveals multiple CYP2E1reductase complexes. Biochemistry 46, 10192-10201 (2007).

52. Larion, M. \& Miller, B. G. Global fit analysis of glucose binding curves reveals a minimal model for kinetic cooperativity in human glucokinase. Biochemistry 49, 8902-8911 (2010).

53. Zhao, H. \& Schuck, P. Global multi-method analysis of affinities and cooperativity in complex systems of macromolecular interactions. Anal. Chem. 84, 9513-9519 (2012).

54. Hamill, O. P., Marty, A., Neher, E., Sakmann, B. \& Sigworth, F. J. Improved patch-clamp techniques for high-resolution current recording from cells and cell-free membrane patches. Pflugers Arch. 391, 85-100 (1981).

\title{
Acknowledgements
}

We thank K. Schoknecht for excellent technical assistance. This work was funded by Project P2 of the Research Unit 2518 of the Deutsche Forschungsgemeinschaft 'DynIon' to K.B.

\section{Author contributions}

C.S. conducted the patch-clamp recordings and analyses, did the molecular biology and prepared the figures. T.E. did the global fit analyses. S.H., E.S. and R.S. participated in programming the software. C.U. contributed to the patch-clamp recordings. F.S. and A.S. designed the fluorescent ATP derivative. F.S. performed the chemical synthesis of the fluorescent ATP derivative. K.B. initiated and designed the study and wrote the manuscript.

\section{Funding}

Open Access funding enabled and organized by Projekt DEAL.

\section{Competing interests}

The authors declare no competing interests.

\section{Additional information}

Supplementary Information The online version contains supplementary material available at https://doi. org/10.1038/s41598-020-78672-w.

Correspondence and requests for materials should be addressed to K.B.

Reprints and permissions information is available at www.nature.com/reprints.

Publisher's note Springer Nature remains neutral with regard to jurisdictional claims in published maps and institutional affiliations.

\begin{abstract}
(c) (1) Open Access This article is licensed under a Creative Commons Attribution 4.0 International C. License, which permits use, sharing, adaptation, distribution and reproduction in any medium or format, as long as you give appropriate credit to the original author(s) and the source, provide a link to the Creative Commons licence, and indicate if changes were made. The images or other third party material in this article are included in the article's Creative Commons licence, unless indicated otherwise in a credit line to the material. If material is not included in the article's Creative Commons licence and your intended use is not permitted by statutory regulation or exceeds the permitted use, you will need to obtain permission directly from the copyright holder. To view a copy of this licence, visit http://creativecommons.org/licenses/by/4.0/.
\end{abstract}

(C) The Author(s) 2020 